\section{SMGrøup}

SM

\section{Musculoskeletal Disorders}

\author{
Article Information \\ Received date: Jun 05, 2017 \\ Accepted date: Sep 25, 2017 \\ Published date: Sep 29, 2017 \\ ${ }^{*}$ Corresponding author \\ Dror Robinson, Department of \\ Orthopedic Surgery, Tel Aviv University, \\ Israel, Tel: +972543342899; \\ Fax: +97289206013; \\ Email: dror61@gmail.com
}

Distributed under Creative Commons CC-BY 4.0

Keywords Ankle fracture; Arthroscopy; Cartilage damage; Post traumatic osteoarthritis; Chondral damage

Article DOI 10.36876/smmd.1022

\title{
Ankle Arthroscopy Findings During Ankle Fracture Fixation and Mid-Term Prognosis
}

\author{
Mustafa Yassin, Avraham Garti, Eyal Heller and Dror Robinson ${ }^{\star}$ \\ Department of Orthopedic Surgery, Tel Aviv University, Israel
}

\section{Abstract}

Anatomic reduction of ankle fractures does not ensure a normal ankle function. This might be related to chondral damage during the fracture or other intra-articular pathology. Arthroscopy in the setting of acute operative management of ankle fractures provides a means to completely assess intra-articular pathology and sometimes even direct therapeutic intervention. To date there is no consensus in the literature regarding the indications for arthroscopy in the setting of ankle fractures. It is our practice to perform an arthroscopic assessment of all ankle fractures requiring surgical intervention. The current study retrospectively evaluated a consecutive series of twenty-two adult patients who underwent routine arthroscopy performed during open reduction and internal fixation of ankle fractures and its correlation with the clinical results at a follow-up of 2 years. The clinical results at 2 year follow-up results demonstrated an average AOFAS score of $82 \pm 8$. Patients with grade 4 talar damage fared significantly worse $(70 \pm 8)$ than both patients with grade 3 talar damage $(83 \pm 7)$ and with normal talus structure during arthroscopy $87 \pm 9(5.42, p<0.001)$.

In conclusion the findings of this limited series seem to indicate that final clinical results at 2 years follow-up correlate with the presence of chondral damage observed during arthroscopy. A randomized clinical trial should be performed in order to assess the possible advantages of ankle arthroscopy as compared with open reduction technique in improving treatment outcomes in ankle fracture fixation.

\section{Introduction}

The well-established technique for treatment of displaced ankle fractures involves open reduction and internal fixation which has been shown to confer some advantage, even in geriatric patients, as compared to conservative treatment [1].

Despite hardware improvement, there remains a substantial group of patients with less than optimal surgical results [2,3]. Many factors might contribute to this limited clinical success, including ligamentous injury, altered proprioception and muscle function [2] as well as intraarticular pathology.

While the routine open exposure technique allows the evaluation of the surfaces to a limited extent, some intra-articular pathology might be missed. This may potentially be solved by performing ankle arthroscopy a technique that allows evaluation of the intra-articular condition. Arthroscopy in the setting of acute operative management of ankle fractures provides a means to completely assess intra-articular pathology and sometimes even direct therapeutic intervention. This minimally invasive approach also might reduce tissue damage during open surgery which in turn could impact clinical outcome. Furthermore, such a technique may potentially improve fracture reduction and detect multiple soft tissue injuries that are undetectable using fluoroscopy [4-7].

Arthroscopic-assisted treatment of ankle fractures has been utilized for over a decade [8] and $>70 \%$ of ankle fractures have chondral damage associated with the ankle fracture, especially in the high energy fracture types [9]. The defect is most often in the talus and certain injury types are more often associated with osteoarthritis during later follow-up [10]. Some authors have suggested that performing an arthroscopy does not affect the final outcome of the intervention [11]. The current retrospective study aimed to assess whether surgical findings during ankle arthroscopy can aid in predicting the eventual prognosis.

\section{Methods}

\section{Patients}

A consecutive series of twenty-two patients with ankle fractures were included (Table 1). The patients were all treated by the same team (D.R. \& E.H.) using locked plates. There were no cases of peri-operative deep infection, a single case of delayed wound healing due to skin blistering and one due to sterid use, and no cases of peri-operative nerve damage.

\section{OPEN ACCESS}


Patients were evaluated pre-operatively and every 3 months, until2 years after surgery, during routine clinic follow-up visits.

Functional outcomes at two years after operation were graded using the AOFAS scoring scales, which ranges from 0 to 100, with higher scores indicating lesser impairment [12].

\section{Syndesmosis stabilization}

Syndesmosis stabilization was performed when necessary using either Tight Rope TM (Arthrex, USA) or two 3-cortical syndesmotic screws. The former option allows immediate weight bearing while the latter requires 6 weeks of non-weight bearing and secondary screw removal.

\section{Arthroscopic Surgical Technique}

All patients were operated using lumbar anesthesia while lying in the supine position.

We avoided the use of a tourniquet to decrease the risk of nerve damage and compartment syndrome. The arthroscopy was performed using standard anteromedial and anterolateral portals using a $2.7 \mathrm{~mm}$ scope. Internal fixation was performed through a minimally invasive approach in some of the cases (12/22) with percutaneous plate insertion and screw fixation, and via a standard open approach in the

Table 1: Patient characteristics and arthroscopy evaluation findings.

\begin{tabular}{|c|c|c|c|c|c|}
\hline \multirow{2}{*}{ Age } & \multirow{2}{*}{ Weber Fracture Type } & \multicolumn{3}{|c|}{ Grade from Arthroscopy Evaluation } & \multirow{2}{*}{ Syndesmosis } \\
\hline & & Talar Cartilage & Tibia Cartilage & Fibular Cartilage & \\
\hline 22 & $\mathrm{~B}$ & norm & norm & 2 & norm \\
\hline 45 & $\mathrm{C} 1$ & 3 & 3 & 3 & Anterior rupture \\
\hline 48 & $\mathrm{C} 2$ & 3 & 4 & norm & complete rupture \\
\hline 33 & $\mathrm{~B}$ & norm & 3 & norm & complete rupture \\
\hline 75 & B & 2 & 3 & norm & complete rupture \\
\hline 69 & $\mathrm{C} 1$ & 4 & 3 & norm & complete rupture \\
\hline 71 & $\mathrm{C} 2$ & 3 & 3 & norm & complete rupture \\
\hline 38 & $\mathrm{C} 2$ & 4 & 3 & norm & complete rupture \\
\hline 81 & A & 3 & norm & norm & Norm \\
\hline 57 & B & norm & norm & norm & Anterior rupture \\
\hline 22 & $\mathrm{C} 2$ & 4 & norm & 2 & complete rupture \\
\hline 34 & $A$ & norm & 3 & norm & Norm \\
\hline 37 & B & 3 & 3 & norm & Anterior rupture \\
\hline 27 & $\mathrm{C} 1$ & 3 & 3 & norm & Anterior rupture \\
\hline 54 & $\mathrm{C} 2$ & 3 & 4 & 2 & complete rupture \\
\hline 47 & $\mathrm{C} 2$ & 3 & 4 & 2 & complete rupture \\
\hline 41 & A & norm & norm & norm & norm \\
\hline 19 & B & 3 & 3 & norm & complete rupture \\
\hline 20 & $\mathrm{C} 1$ & 3 & 3 & norm & complete rupture \\
\hline 28 & $\mathrm{C} 2$ & 4 & norm & norm & complete rupture \\
\hline
\end{tabular}

Norm, no apparent damage visually or by palpation; Anterior rupture, rupture of the anterior syndesmosis which presents like an opened door whose hinges are on the posterior ligaments.

Citation: Yassin M, Garti A, Heller E and Robinson D. Ankle Arthroscopy Findings During Ankle Fracture Fixation and Mid-Term Prognosis. SM Musculoskelet Disord. 2017; 2(3): 1022

https://dx.doi.org/10.36876/smmd.1022 
cartilage damage was present in less than one third of the cases (28\%). In $(6 / 22) 36 \%$ of the patients with grade 4 cartilage damage, a loose body was removed Most of the patients (78\%) had some syndesmosis damage. In 4/22 patients hypertrophic synovitis of the anterior ankle was observed and resected.

In general, 2 years follow-up results were quite good with an average AOFAS score of $82 \pm 8$. Patients with grade 4 talar damage were doing significantly worse $(70 \pm 8)$ than patients with grade 3 talar damage (83 \pm 7 ) and those with normal talus during arthroscopy $87 \pm 9$ (ANOVA, F-statistic 5.42, $\mathrm{p}<0.001$ ).

Patients with syndesmosis damage $(80 \pm 8)$ tended to not do as well as those with normal syndesmosis $(88 \pm 7)$ but the difference was not statistically significant (Student's t-test, t-value $-1.8, \mathrm{p}<0.085$ ).

\section{Discussion}

Arthroscopic evaluation of the ankle joint during fracture fixation appears to allow appreciation of the actual damage as well as treatment of loose bodies. In the current study no attempt was made to treat any observed chondral lesions using sophisticated marrow-stimulation or scaffold techniques. Thus, it is unclear whether the worse prognosis could be mitigated by induction of cellular repair at the time of fracture fixation. In the current study the clinical outcome show no arthroscopy related complications. Compartment syndrome, is a known risk of arthroscopy during fracture fixation [13]. To reduce the risk of this unfortunate complication we avoided using a fluid infusion pump and relied solely on gravity-assisted irrigation.

The high frequency of chondral damage occurring during ankle fractures has been described by many authors $[8,9]$. This study demonstrates that cartilage damage observed during arthroscopy affects the long term prognosis as evaluated using the AOFAS score. This supports the findings of Stufkens et al [10]. Who observed progression to osteoarthritis in patients with talar injury or medial malleolar cartilage damage. Previous authors have found that unimalleolar fractures do not affect the gait pattern as severely as bimalleolar and trimalleolar fractures [2].This observation is probably related to the amount of cartilage damage in the latter types.

Limitations of the study include a small cohort, and a relatively short follow-up period.

In conclusion, this study adds to the growing body of evidence indicating the clinical benefits of performing ankle arthroscopy on ankle fractures in adults, and the positive impact this minimallyinvasive and revealing technique has on patient outcomes.
Further research should be carried out in order evaluate whether using a bone-marrow stimulation technique or a scaffold might allow better prognosis for patients with acute fractures and associated cartilage damage.

\section{References}

1. Bariteau JT, Hsu RY, Mor V, Lee Y, DiGiovanni CW, Hayda R. Operative versus nonoperative treatment of geriatric ankle fractures: a medicare part a claims database analysis. Foot Ankle Int. 2015; 36: 648-655.

2. Segal G, Elbaz A, Parsi A, Heller Z, Palmanovich E, Nyska M, Feldbrin Z, Kish B. Clinical outcomes following ankle fracture: a cross-sectional observational study. J Foot Ankle Res. 2014; $7: 50$.

3. Tejwani NC, Park JH, Egol KA. Supination external rotation ankle fractures: A simpler pattern with better outcomes. Indian J Orthop. 2015; 49: 219-222.

4. Abdel-Hamid MZ, Chang $\mathrm{CH}$, Chan YS, Lo YP, Huang JW, Hsu, KY, et al. Arthroscopic evaluation of soft tissue injuries in tibial plateau fractures: retrospective analysis of 98 cases. Arthroscopy. 2006; 22: 669-675.

5. Chan YS. Arthroscopy- assisted surgery for tibial plateau fractures. Chang Gung Med J. 2011; 34: 239-247.

6. Hung SS, Chao EK, Chan YS, Yuan LJ, Chung PC, Chen CY, et al. Arthroscopically assisted osteosynthesis for tibial plateau fractures. J Trauma. 2003; 54: 356-363.

7. Lemon RA, Bartlett DH. Arthroscopic assisted internal fixation of certain fractures about the knee. J Trauma. 1985; 25: 355-358.

8. Leontaritis N, Hinojosa L, Panchbhavi VK. Arthroscopically detected intraarticular lesions associated with acute ankle fractures. J Bone Joint Surg Am. 2009; 91: 333-339.

9. Aktas S, Kocaoglu B, Gereli A, Nalbantodlu U, Guven O. Incidence of chondral lesions of talar dome in ankle fracture types. Foot Ankle Int. 2008; 29: 287-292.

10. Stufkens SA, Knupp M, Horisberger M, Lampert C, Hintermann B. Cartilage lesions and the development of osteoarthritis after internal fixation of ankle fractures: a prospective study. J Bone Joint Surg Am. 2010; 92: 279-286

11. Thordarson DB, Bains R, Shepherd LE. The role of ankle arthroscopy on the surgical management of ankle fractures. Foot Ankle Int. 2001; 22: 123-125.

12. Paluvadi SV, Lal H, Mittal D, Vidyarthi K. Management of fractures of the distal third tibia by minimally invasive plate osteosynthesis - A prospective series of 50 patients. J Clin Orthop Trauma. 2014; 5: 129-136.

13. Imade S, Takao M, Miyamoto W, Nishi H, Uchio Y. Leg anterior compartment syndrome following ankle arthroscopy after Maisonneuve fracture. Arthroscopy. 2009; 25: 215-218. 